\title{
ELECTRONIC TRANSPORT IN METALLIC FILMS - A TOOL FOR SCANNING TUNNELING MICROSCOPY INVESTIGATIONS
}

\author{
G. Reiss, H. Brückl \\ Institut für Angewandte Physik III, Universität Regensburg, \\ Universitätsstr. 31, D - 8400 Regensburg
}

(Received 19 May 1991)

\begin{abstract}
In this contribution, we show, that the evaluation of electronic transport parameters in confined systems can be considerably improved by additional Scanning Tunneling Microscopy (STM) imaging of the limiting surfaces. The thin films which are experimentally available usually show both a mesoscopic and a microscopic (i.e. atomic) surface roughness. These two roughnesses, however, are of well separated magnitudes and therefore can be treated either by classical averaging or by quantum mechanics. In order to ensure reliable STM results, the resolution especially of mesoscopic surface features will be discussed. Provided reasonable STM resolution, the experimental data for the resistivity can be interpreted for the first time with a realistic, two dimensional model for the current transport in thin films. Forthcoming applications concerning the distribution of the potential related to current transport in thin films will be discussed.
\end{abstract}

\section{Introduction}

The surface scattering of the conduction electrons considerably enhances the resistivity of thin metallic films. Whereas quantum mechanical considerations of the correlation of the scattering and the surface roughness have been developed in the last years $^{(1-4)}$, the real surface structures have not yet been introduced into the interpretations of experimental data. One dimensional models ${ }^{(5,6)}$, for example, treated the roughness with a sinusoidal approximation. This, however, clearly oversimplifies the problem. Therefore, the accuracy of the transport parameters obtained from these models seems to be very moderate. Usually, thin metallic films exhibit two roughnesses of well separated magnitudes: A mesoscopic roughness of the order of some $\mathrm{nm}$ 's caused by the polycrystalline structure and a microscopic roughness due to, for example, atomic steps or dislocations. Whereas the first one can be treated using a fluctuating film thickness $^{(4,6,7)}$, the second gives rise to electronic surface scattering and can be described by a scattering strength of the surface ${ }^{(1,4)}$. The interpretation of the thickness dependence of the conductivity thus can be considerably improved by using realistic surface profiles.

\section{Surface Topography by STM}

Experimentally, the surfaces can be directly imaged using the capabilities of STM: This method offers both atomic as well as mesoscopic resolution. Whereas, however, the first one can be even obtained with blunt tips, the imaging of mesoscopic surface structures claims a critical estimation of the obtained height resolution: Clearly, the resolution is limited by the front shape of the tunneling tip. Deep and narrow corrugations of the surface may not be reflected in the measured STM image. The amount of these unresolved parts of the surface, however, can be estimated by a deconvolution of the STM image using an appropriate tip shape ${ }^{(8)}$. The vertical resolution can be estimated from this amount of unresolved, i.e. 'black-hole' surface area $A_{b}{ }^{(9)}$. The height resolution drastically depends on $A_{b}$ : If the amount of black-holes exceeds about $5 \%$ of the whole surface, the vertical resolution can be supressed to $50 \%$ of the real surface roughness ${ }^{(9)}$. 
Therefore, especially concerning the introduction of STM results in a quantitative treatment of physical thin film properties, the depth resolution must be guaranted for each individual image.

In case of reasonable resolution, STM provides a reliable function $i(x, y)$ describing the two dimensional surface profile. The introduction of this function into the treatment of the thin film conductivity will be discussed in the next section.

\section{Conductivity of Thin Films}

Theoretical discussions of the conductivity of thin films have been given, for example, by Tešanović and coworkers ${ }^{(1)}$. They introduced the surface roughness as spatially fluctuating confining potential, which gives rise to scattering of the conduction electrons. The result of the perturbational treatment of this problem is given in eq. (1):

$$
\begin{gathered}
\sigma_{l o c}\left(d(x, y), l_{\infty} \cdot h^{2}, \sigma_{\alpha}\right)= \\
\frac{\sigma_{\infty}}{n_{c}} \cdot \sum_{n=1}^{n=n_{c}}\left(1+\frac{l h^{2} k_{F}^{2}}{6 \pi d(x, y)} \cdot\left(\frac{n}{n_{c}}\right)^{2}\right)^{-1}(1)
\end{gathered}
$$

Here, $\sigma_{\infty}$ is the conductivity of bulk material, $l_{\infty}$ is the intrinsic mean free path, $h$ is the microscopic rms roughness of the surface potential, $n_{c}$ is the number of occupied subbands and $k_{F}$ is the Fermi wavevector.

The local thickness $d(x, y)$ is supplied by the STM image. This function can be found from $i(x, y)$ by evaluating the mean surface height ${ }^{(9)}$ and identifying it with the mean film thickness $d_{m}$. The task of STM thus is the determination of the surface topography for different thicknesses. For the structure of the surface may change with increasing film thickness, measurements must be performed for different stages of the film growth. A typical example for the surface topography of a $20 \mathrm{~nm}$ thick $\mathrm{Ni}$ film is shown in fig. 1.

The surface of these films is strongly structured. The mean roughness amounts to $3.5 \mathrm{~nm}$. The maximum deviation from the mean surface height is $6 \mathrm{~nm}$. These values remain constant for thicknesses between $10 \mathrm{~nm}$ and $40 \mathrm{~nm}$. Thus this surface profile can be used for fitting eq.(1) to the experimental data of the thickness dependence of the conductivity. In order to perform realistic, two dimensional calculations, however, the film must be modeled as a resistor network. The conductance of each resistor is given by the local thickness and eq.(1). From the conductances, the distribution of the potential $\mathbf{V}(\mathbf{x}, \mathbf{y})$ can be calculated self-consistently by Kirkpatrick's for mula(10):

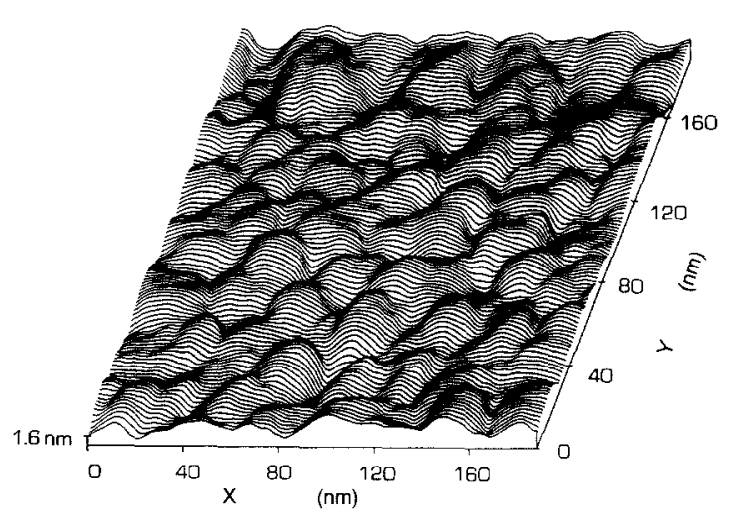

Fig. 1: Typical STM-image of a $20 \mathrm{~nm}$ thick Ni film evaporated on glass at room temperature.

80<smiles>CC(C)C</smiles>

$\sum_{\substack{\bar{D} \\ \mathbb{D}}}^{\mathbb{2}} 40$

20

$\begin{array}{llll}0.04 & 0.08 & 0.12 & 0.16 \\ & 1 / \text { Film-thickness } & \left(\mathrm{nm}^{-1}\right) & \end{array}$

Fig. 2: The resistivity of thin Ni films vs.the reciprocal film thickness. Line: Experimental results. Crosses: One dimensional model. Squares: two dimensional model.

$$
V_{i}=\sum_{j=1}^{4} V_{j} g_{i j} \cdot\left(\sum_{j=1}^{4} g_{i j}\right)^{-1}
$$

The sum includes the nearest neighbors $\{j\}$ of the node $i$ of the network and $g_{i j}$ are the local conductances between node $i$ and node $j$. Here, a $128 \times 128$ grid has been used. Note, that this formalism holds, as long as the lateral dimensions of the surface corrugations are much larger than their height. From the distribution of the potential, the current flowing through the film can be estimated. Using these values, the theory can be fitted to the experimental curves by a variation of $\sigma_{\infty}$ and the product $I_{\infty} h^{2}$. Fig. 2 shows the result of this calculation: 


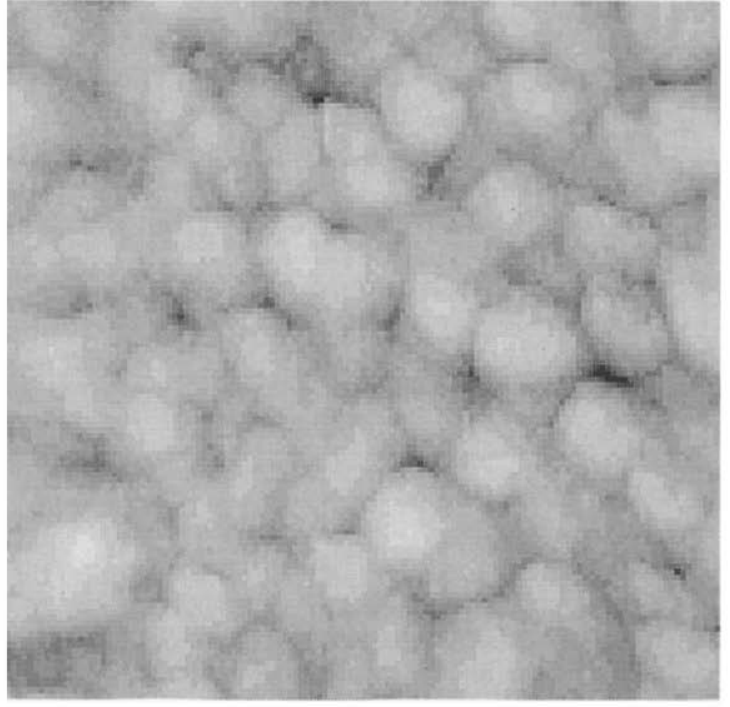

Fig.3a: Greyscale topography of a $10 \mathrm{~nm}$ thick $\mathrm{Ni}$ film (image size $200 \mathrm{~nm} \times 200 \mathrm{~nm}$ ). The scale is $10 \mathrm{~nm}$ from dark to white.

From the one dimensional calculation, the following parameters are obtained: $\rho_{\infty}=19.5 \mu \Omega \mathrm{cm}$ and $I_{\infty} h^{2}=1.25 \mathrm{~nm}^{3}$. The more realistic, two dimensional treatment gives a larger bulk resistivity and larger values of $\mathrm{I}_{\infty} \mathrm{h}^{2}: \rho_{\infty}=21 \mu \Omega \mathrm{cm}$ and $\mathrm{I}_{\infty} \mathrm{h}^{2}=1.4 \mathrm{~nm}^{3}$. Using the Drude-Sommerfeld formula, the possible range for the microscopic roughness can be estimated: $0.2 \mathrm{~nm}<\mathrm{h}<0.45 \mathrm{~nm}$. For $h$ should be of the order of the Fermi wavelength, this check demonstrates the physical reliability of the evaluated transport parameters.

\section{Further Applications}

The problem of current transport in thin films is related to several topics: For rough films, the related potential will be distributed inhomogeneously as soon as the thickness becomes comparable to the intrinsic mean free path. Therefore, the gradient of the potential, i.e. the local field, will be strongly enhanced at locations with small local thickness. Using the formalism discussed in this contribution, the two dimensional distribution of the potential can be calculated. Fig's.3a and 3b show the topography ( $3 a$ ) and the corresponding magnitude of the local field (3b) for a $10 \mathrm{~nm}$ thick Ni film. The local conductances used in eq. (2) have been evaluated using the parameters of fig. 2 :

From the deconvolution ${ }^{(8)}$ of the STM image of fig.3a, the amount of 'black holes' can be estimated to be smaller than $3 \%$ of the whole

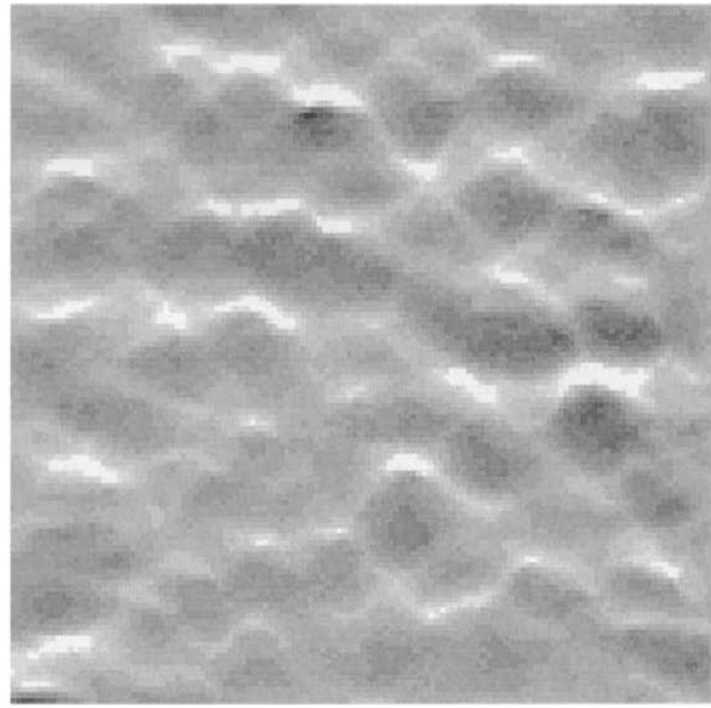

Fig.3b: Distribution of the local field on the film shown in fig. 3a. White corresponds to a field twice as large as dark.

surface area. The vertical resolution thus is larger than $95 \%{ }^{(9)}$. Therefore, the topography of fig. $3 a$ can be used for the calculation of the current related distribution of the local electrostatic field ${ }^{(10)}$. Here, the direction of the current was chosen from the left to the right side of fig. $3 a$. The result shown in fig. $3 \mathrm{~b}$ agrees very well with the topography of fig.3a: The valleys directed almost perpendicular to the current correspond to strongly enhanced values of the local field, i.e. large drops of the local potential due to the lower conductances in these regions.

Therefore, fig's.3a and 3b demonstrate, that for thin, rough films the current related distribution of the electrostatic field can be expected to be rather inhomogeneous and correlated with the local topography. Experimentally, similar results have been obtained by Scanning Tunneling Potentiometry ${ }^{111}$. This confirms, that the spatially varying conductance must be taken into account in order to obtain realistic interpretations of the transport properties.

\section{Conclusions}

In summary, a new, two dimensional treatment of the electronic transport in thin films has been discussed. Using a combination of in-situ measurements of the resistivity and STM imaging of the surface, physically reliable transport parameters can be obtained by fitting the theoretical expressions to the experimental curves. 
Moreover, this method has additional possible applications: Potentiometric STM measurements often show a stepped distribution of the potential for thin films carrying current. This can be confirmed by corresponding calculations of the current related distribution of the electrostatic field in these structures. The results show inhomogeneous but non-statistical variations of the local electric field which are strongly correlated with the topography. Clearly, the results discussed in this contributions are of relevance for several problems of the current transport in thin films as, for example, electromigration or the temperature dependence of the resistivity. The relation of the present discussion with the temperature dependent transport properties will be discussed elsewhere ${ }^{(12)}$.

\section{Acknowledgements}

We are indepted to J.Vancea for valuable discussions, to F.Schneider for STM software and to H. Hoffmann for his support of this work.

\section{REFERENCES}

1. Z.Tešanovic, M.V.Jaric and S.Maekawa, Physical Review Letters 57, 2760 (1986)

2. D.Calecki and G.Fishman, Surface Science 229, 110 (1990)

3. C.S.Chu and R.S.Sorbello, Physical Review B 38, 7260 (1988)

4. N.Trivedi and N.W.Ashcroft, Physical Review B 38, 12298 (1988)

5. K.Fuchs, Proceedings of the Cambridge Philosophical Society 34, 100 (1938)

6. Y.Namba, Japanese Journal of Applied Physics 9, 1326 (1970)

7. G.Reiss, E.Hastreiter, H.Brückl and J.Vancea Physical Review B 43, 5176 (1990)

8. G.Reiss, F.Schneider, J.Vancea and H.Hoffmann, Applied Physics Letters 57, 867 (1990)

9. G.Reiss, H.Brückl, J.Vancea, R.Lecheler and E. Hastreiter, Journal of Applied Physics 70, 523 (1991)

10. S.Kirkpatrick, Review of Modern Physics 45 , 574 (1973)

11. J.P.Pelz and R.H.Koch, Review of Scientific Instruments 60, 301 (1989)

12. G.Reiss and H.Brückl, unpublished 